\title{
THE GRANULAR STRUCTURE OF SNOW: AN INTERNAL-STATE VARIABLE APPROACH
}

\author{
By' A.C. HANSEN \\ (Department of Mechanical Engineering, University of Wyoming, Laramie, Wyoming 82071, U.S.A.) \\ and R.L. BROWN
}

(Department of Civil Engineering and Engineering Mechanics, Montana State University,

Bozeman, Montana 59717, U.S.A.)

\begin{abstract}
A statistical model characterizing the granular structure of snow is developed using quantitative stereology. The model is based on specific parameters (e.g. bond radius, grain-size, etc.) which take the form of internal-state variables in a constitutive theory for high-rate deformation of snow. In addition to parameters developed by other authors in previous investigations, a new parameter characterizing the mean bond length is developed. More significantly, general relations are derived for the mean number of bonds per grain and mean number of grains per unit volume without making any assumptions regarding the shape or size of the ice grains, or their respective distributions.
\end{abstract}

RÉSUMÉ. La structure granulaire de la neige: une approche avec les variables d'état interne. On développe un modèle statistique pour caractériser la structure granulaire de la neige à l'aide d'une stéréologie quantitative. Le modèle est basé sur des paramètres spécifiques (comme le rayon de voisinage, la taille du grain, etc.) qui prend en compte la forme des variables d'état interne dans une théorie constitutive pour de fortes vitesses de deformation de la neige. En plus des paramètres utilisés par d'autres auteurs lors de précédentes recherches, on introduit un nouveau

\section{INTRODUCTION}

The mechanical properties of snow under high strainrates and finite deformations are assumed to be adequately determined by an elastic, rate-sensitive plastic representation of the material. One approach that falls under this category is based on non-equilibrium thermodynamics with internalstate variables. This type of formulation readily incorporates parameters reflecting the microstructure of the snow cover through the state variables, while at the same time remaining essentially a continuum theory.

In what follows, a structural model for snow is developed based on a set of nine internal-state variables which describe the microstructure of snow at the granular level. The variables are developed on the basis of quantitative stereology of surface sections. The necessary assumption which allows results from one section plane to give threedimensional information is randomness of location and orientation of the structural features of the threedimensional network. Kry (1975) has shown that this is satisfied to within $\pm 10 \%$ even after $30 \%$ uniaxial plastic deformation.

\section{SECTION OF THE INTERNAL-STATE VARIABLES}

The internal-state variables must describe the current microstructural state of the material and be capable of representing average measures of the structural rearrangements taking place within the snow cover. The paramètre caractérisant la distance moyenne de voisinage. Enfin, on en déduit des relations générales pour le rayon moyen de voisinage par grain et le nombre moyen de grains par unité de volume sans faire aucune hypothèse au sujet de la forme ou taille des grains de glace, ni de leur répartitions respectives.

ZuSAMMENFASSUNG. Die Kornstruktur von Schnee: Eine Lösung über innere Zustandsvariable. Mit Hilfe der quantitativen Stereologie wird ein statistisches Modell zur Charakterisierung der Kornstruktur des Schnees entwickelt. Das Modell stützt sich auf spezifische Parameter (z.B. Bindungsradius, Korngrösse, etc.), welche die Form innerer Zustandsvariabler in einer konstitutiven Theorie für hochgradige Verformung von Schnee annehmen. Zusätlich zu Parametern, die von anderen Autoren in früheren Untersuchungen herangezogen wurden, wird ein neuer Parameter eingeführt, der die mittlere Bindungslänge kennzeichnet. Von noch grösserer Bedeutung ist die Herleitung allgemeiner Beziehungen für die mittlere $\mathrm{Zahl}$ von Bindungen pro Korn and von Körnern pro Volumeneinheit, die ohne Voraussetzungen hinsichlich der Form oder Grösse der Eiskörner oder deren jeweiliger Verteilung vorgenommen wird.

variable selection process attempted to include the most significant aspects of the granular structure and the dynamic environment of the material under high-rate deformation.

The variables chosen should be able to characterize the dominant deformation mechanisms such as bond fracture, intergranular glide, and neck growth at the bonds. Furthermore, they must account for various phenomena known to occur under compressive loadings such as:

(1) The effects of pore pressure,

(2) A locking mechanism for grains during intergranular glide,

(3) Coupling of the deviatoric and volumetric responses,

(4) Work hardening, and

(5) Local inertial effects.

Finally, the state variables must be chosen so as to make measuring them a feasible task.

Before proceeding with the selection of the state variables, it is necessary to clarify the notation to be used. When discussing the behavior of dry snow in the general macroscopic sense, the material is considered to be a multiphase mixture consisting of an ice phase and an air phase. Each phase is denoted by a subscript as follows: a, air phase; i, ice phase. This convention is in accordance with that used in mixture-theory studies of mechanical properties of snow. 
An alternative approach will be used when measuring the internal-state variables in the snow at the granular level. Here, the snow is considered to be a three-phase material consisting of an ice-grain phase, ice-neck phase, and air phase. The three phases are denoted by the following Greek subscripts: $\alpha$, ice-grain phase; $\beta$, ice-neck phase; and $\gamma$, air phase.

In the following discussions, no attempt is made to state which of the two previous approaches is being used since the subscripts are self-explanatory. Finally, in quantitative stereology, it is necessary to differentiate between length, area, and volume measurements. Therefore, let the following capitalized subscripts denote this difference: L, length measurement; A, area measurement; and $\mathrm{v}$, volume measurement.

Taking into account the desired properties of the state variables, the following parameters were chosen to formulate the statistical model for snow. A discussion of their significance and measurability is given later.

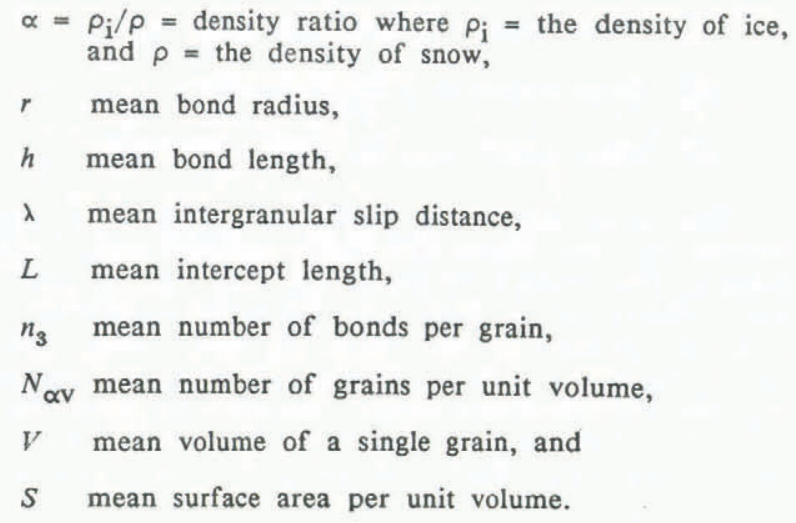

\section{MEASURING THE STATE VARIABLES}

Before discussing each internal-state variable, it is necessary to describe the stereological measurements which are required to obtain the desired information. As mentioned previously, snow at the granular level is considered to be a three-phase material consisting of ice grains, ice necks, and air. Kry (1975) developed an operational definition to identify grain bonds in a twodimensional surface section. If a grain bond is cut by a section plane, it will appear as a line connecting opposite edges of the ice. Three criteria are required to identify these bonds:

(1) A minimum constriction must exist; a $30 \%$ constriction on the plane is used as a cut-off.

(2) Both edges of the ice must show the constriction.

(3) The notches on each edge must point approximately towards each other.

The neck region of a grain bond is defined as that area surrounding the bond where the grain goes from convex to concave with respect to the outward normal. The bond is defined as the region of minimum constriction. Figure 1 shows an image-enhanced snow sample which delineates the three phases of interest. Using the enhanced image, the following measurements can now be made. The code contained within the parentheses indicates the method of analysis, e.g. visual count (VC) or computerized image analysis (IA).

$P_{\alpha} \quad$ Area fraction of points falling in the ice-grain phase (IA).

$P_{\beta} \quad$ Area fraction of points falling in the ice-neck phase (IA).

$P_{y} \quad$ Area fraction of points falling in the air phase (IA).

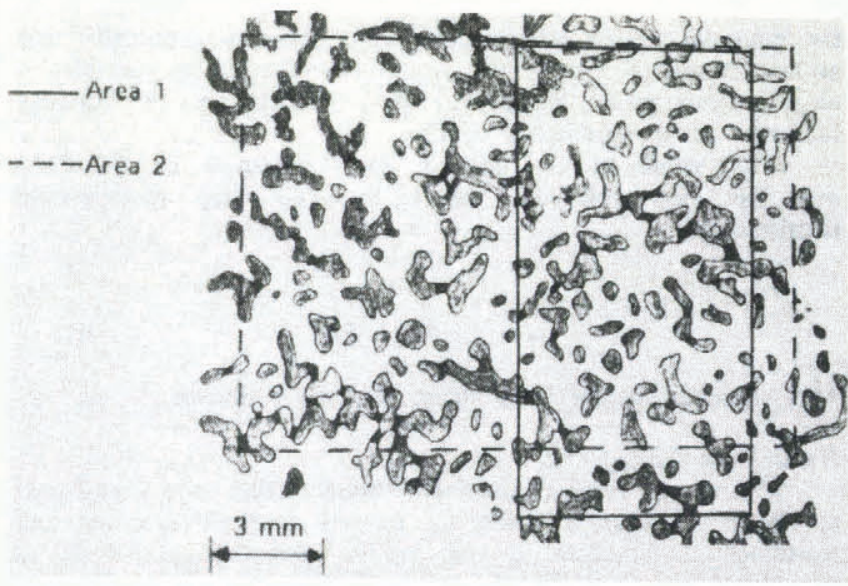

Fig. 1. Image-enhanced surface section showing the three phases of interest. The solid and broken lines indicate respective areas of analysis.

$\bar{E}=\overline{\left(1 / d_{2}\right)}$ Harmonic mean of lines representing grain bonds in the surface section (IA). Hence, $d_{2}$ is defined as the two-dimensional bond diameter.

$N_{\alpha \mathrm{L}} \quad$ Number of interceptions of ice grains in the microstructure per unit length of a random test line (IA) (see Fig. 2).

$N_{\alpha} \quad$ Number of grains in the test area (VC).

$N_{B} \quad$ Number of bonds in the test area (VC).

$f_{2}(n) \quad$ Probability distribution of the number of bonds per grain cut by the section plane (VC).

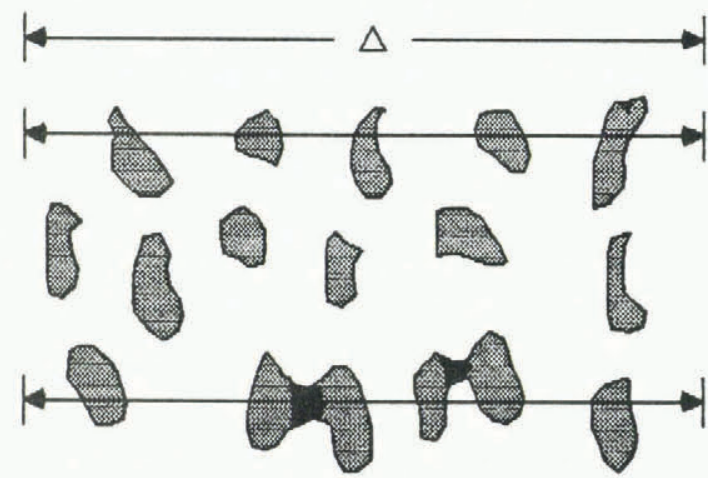

(a)

Fig. 2. Counting relationship for $N_{\propto \mathrm{L}}$ (a) $N \propto \mathrm{L}=5 / \Delta, \quad(b$
$N_{\alpha \mathrm{L}}=6 / \Delta$.

It should be noted that all measurements which require a visual count can be performed on the order of minutes. The time-consuming part of the analysis will be in the area of image enhancement.

At this point one can proceed with a discussion of each of the state variables.

Density ratio $(\alpha)$

The density ratio is defined by the following expression:

$$
\alpha=\rho_{\mathrm{i}} / \rho .
$$

The density of snow has been the primary measure of the "state" of the material in many of the past investigations of the mechanical behavior of snow. This is primarily because of the ease and quickness of density measurement in the field. However, recent papers (Gubler, 1978) have shown that density alone cannot adequately describe the state of 
the material under certain conditions. This is especially true at low densities where the impact of other state variables is more significant. However, the importance of density increases as $\alpha$ approaches unity.

The value of the density ratio obtained in the field may be cross checked using the following stereological relationship:

$$
\alpha=1 /\left(P_{\alpha}+P_{\beta}\right) .
$$

This equation neglects the mass of the air phase.

\section{Mean bond radius ( $r$ )}

The mean three-dimensional bond radius is a significant parameter of pressure sintering as well as bond strength and resistance to fracture. Brown (1979) used this parameter in a volumetric constitutive law based on neck growth. Fullman (1953) derived the necessary relations with the assumption that the grain bonds are circular disks. Kry (1975) has shown that this idealization yields self-consistent results.

The governing equation for the mean bond radius as derived by Fullman is

$$
r=\pi /(4 \bar{E}) .
$$

\section{Mean bond length}

The mean bond length is also a significant parameter for modeling pressure sintering. This was used in Brown's "neck-growth" volumetric constitutive law. However, until now the authors are unaware of any attempts to evaluate this parameter stereologically. The statistical model of bond length is developed by idealizing the necks of grains to be cylinders. This is based on the fact that self-consistent results have been obtained by idealizing the grain bonds as disks. Clearly, the neck regions of an ice grain are not perfectly cylindrical. However, the definition still provides a meaningful relationship when comparing differing types of snow in that it is a consistent measure of bond length. Fullman (1953) has derived a relationship for the number of bonds per unit volume based on knowledge of the number of bonds per unit area. This relationship is

$$
N_{B v}=8 \bar{E} N_{B A} / \pi^{2}
$$

where $N_{B_{\mathrm{A}}}$ is the number of bonds per unit area. Underwood (1970) has shown that a statistically exact equation for determining the volume fraction of the $\alpha$ th constituent is given by

$$
V_{\alpha}=P_{\alpha}
$$

Therefore, by introducing the neck region of an ice grain as a separate phase, one can determine the volume fraction of the necks. The idealized bond length can then be determined by the following expression:

$$
h=P_{\beta} /\left(N_{B \mathrm{v}} \pi r^{2}\right) .
$$

\section{Mean intergranular slip distance $(\lambda)$}

The mean intergranular slip distance is of major importance for characterizing intergranular glide. As this distance decreases, pressure sintering becomes more dominant in the deformation process. Furthermore, this parameter must be inherently related to the locking phenomenon of intergranular glide. The stereological relationship as defined by Fullman (1953) is given by

$$
\lambda=(\alpha-1) /\left(\alpha N_{\alpha L}\right) \text {. }
$$

This equation is valid regardless of size, shape, or the respective distributions of the grains.

\section{Mean intercept length $(L)$}

The mean intercept length provides a measure of the grain-size and, as a result, complements the mean intergranular slip distance. The combination of these two parameters should provide a physical basis for determining a critical "locking" density for grains during intergranular glide. Furthermore, pressure sintering of neck regions will cause changes in $L$.

The mean intercept length as derived by Underwood (1970) is given by

$$
L=\left(P_{\alpha}+P_{\beta}\right) N_{\alpha L}
$$

Again, this equation makes no assumptions about the shape, size, or distribution of the grains.

\section{Remaining state variables}

The remaining internal-state variables must be discussed together because of their close dependence on each other. The variables are given by

(i) Number of grains per unit volume $\left(N_{\propto v}\right)$.

(ii) Mean number of bonds per grain $\left(\bar{n}_{3}\right)$.

(iii) Mean volume of a single grain $(V)$.

(iv) Mean surface area per unit volume $(S)$.

The mean number of bonds per grain is a strong measure of the degree of grain mobility and fracture strength. The number of grains per unit volume and the mean volume of a grain are important parameters for modeling intergranular glide including local inertial effects. Finally, the mean surface area per unit volume is extremely important for computing energy absorption by snow during high-rate deformation. Under high strain-rates, the snow will undergo brittle fracture at the grain bonds. This causes a significant increase in the surface area which in turn is directly related to the free energy.

Until recently, no method was available for determining the mean number of bonds per grain from a section plane. Gubler (1978) derived a technique for determining this value based on comparing a theoretical distribution of the number of bonds per grain, $f_{2}(n)$, seen in a section plane with the actual distribution. This paper appears to be a pioneering effort in this area. The technique involved calculating the probability, $p$, that if a grain with a coordination number $n=1$ is cut by a section plane, its bond also appears in the section plane. The bond areas are assumed to be random and isotropically distributed on the grain surface. Furthermore, the bonds are considered to be small compared to the grain surface. This is consistent with Kry's (1975) definition of a bond.

Unfortunately, Gubler's approach contains one significant weakness in that the stereological relationship used to obtain the number of grains per unit volume is based on all grains having the same size and shape. This is hardly the case for alpine snow, as highly faceted crystals as well as rounded grains appear throughout the snow cover in many sizes.

The problem of accurately determining the number of grains per unit volume from surface sections for a collection of particles of arbitrary size and shape is extremely difficult. Most authors have assumed the particles to be all one size and shape for which many solutions are known. Some solutions are also known for particles of one shape which obey a log-normal distribution in size. DeHoff $(1964,1965)$ has provided solutions for this case for some simple shapes. Finally, Hilliard (1968) has developed a technique for determining $N_{\text {v }}$ for an arbitrary collection of sizes and shapes provided the relative frequency of the various shapes is known. Unfortunately, this theory is unable to produce specific results since shape factors necessary to apply the theory have not been developed.

The approach taken here to determine $N_{\propto y}$ will avoid any specific identification of grain shape other than to obtain a first guess, as this is virtually impossible for alpine snow. The formulation follows Gubler's approach with some notable exceptions.

To begin, the probability of finding a grain with a coordination number between $n$ and $n+d n$ as derived by Gubler is given by 


$$
f_{3}(n)=N_{3} n^{3 i-1} \exp \left(-\xi n^{2 i}\right)
$$

where $n$ is the three-dimensional coordination number, $N_{3}$ is a normalizing constant, and $i$ is a free parameter of the distribution,

and

$$
\xi=\frac{2}{\pi} \frac{1}{n_{3}} \mathrm{r}\left(\frac{3}{2}+\frac{1}{2 i}\right)^{2 i} \text {. }
$$

The normalizing constant may be determined numerically by requiring

$$
\sum_{n=1}^{12} f_{3}(n)=1
$$

This is based on the premise that each ice grain in a snow cover has at least one bond and at most 12 bonds, as in the case for ice.

The resulting two-dimensional probability distribution as derived by Gubler is

$$
f_{2}(1)=N_{2} \sum_{k=1}^{12}\left[\begin{array}{l}
K \\
l
\end{array}\right] p(1-p)^{K-1} f_{3}(k)
$$

where $p$ is defined as the probability that, if a grain with a coordination number $n=1$ is cut by a section, its bond also appears in the section, and $N_{2}$ is a normalizing constant. The normalizing constant $\mathrm{N}_{2}$ does not appear in Gubler's work. The reason for inserting this is as follows. Equation (11) without $N_{2}$ represents a binomial distribution of the probability $p$ multiplied by the three-dimensional probability distribution $f_{3}(k)$. Therefore, the following equality holds:

$$
\sum_{l=0}^{12} f_{2}(1)=1 .
$$

However, when cutting a section of ice with grains having a coordination number of 12 , it is physically possible to cut at most six bonds. Hence, one can require

$$
\sum_{l=0}^{6} f_{2}(1)=1 \text {. }
$$

The probability $p$ is given by the following (Hilliard, 1968):

$$
p=s_{B} / s_{k}
$$

where

$$
\begin{aligned}
& s_{\beta}=\pi N_{\alpha \mathrm{L}} b / N_{\alpha \mathrm{A}}, \\
& s_{k}=4 n_{\alpha \mathrm{L}} / N_{\alpha \mathrm{V}},
\end{aligned}
$$

and

$$
b=2\left(r^{2}-\left(d_{2} / 2\right)^{2}\right)^{1 / 2} .
$$

Notice that $p$ is a function of the number of grains per unit volume, $N_{\alpha v}$. It is at this point where all previous investigations assume specific shape and size distributions and where this theory deviates.

Underwood (1970) provided a table for determining $N_{\alpha v}$ for particles of one size and shape. This is the approach followed by Gubler. The general equation is given by

$$
N_{\alpha \mathrm{V}}=C\left(N_{\alpha \mathrm{A}}^{2} / N_{\alpha \mathrm{L}}\right)
$$

where $C$ is a coefficient depending only on shape. It is interesting to note that $C$ takes on a fairly narrow range of values for a wide variety of shapes, typically ranging from 0.4 to 0.8 for shapes pertinent to alpine snow. Therefore, one can use Equation (15) to obtain a first estimate of
$N_{\alpha y}$. This in turn fixes the probability, $p$, as defined in Gubler's paper.

Now recall an earlier relationship derived by Fullman (1953) for the number of bonds per unit volume

$$
N_{B \mathrm{~V}}=8 \bar{E} N_{\mathrm{BA}_{\mathrm{A}}} / \pi^{2} \text {. }
$$

The above equation is based on the assumption that the bonds may be considered to be a polydispersed system of thin disks. This is precisely what has been assumed throughout this paper. Using this result, the mean number of bonds per grain is then fixed by the equation

$$
\bar{n}_{3}=2 N_{\beta \mathrm{V}} / N_{\propto \mathrm{v}} \text {. }
$$

By neglecting this equation and allowing $\bar{n}_{3}$ to vary, Gubler has an underconstrained system. This could lead to uniqueness problems and produce erroneous results.

By invoking Equation (16), the parameters $N_{\alpha v}$ and $i$ are varied rather than $\bar{n}_{3}$ and $i$ to determine the proper theoretical density distribution $f_{2}(n)$. Hence, a method has been developed for determining $N_{\propto v}$ without specific consideration of the individual grain shapes and sizes other than to obtain a first guess.

Once $N_{\alpha v}$ is determined, the mean volume of a single grain including the "necks" is given by

$$
V=\left(P_{\alpha}+P_{\beta}\right) / N_{\alpha v} .
$$

The neck regions in this variable are included since the neck is assumed to become an integral part of the grain when a bond fractures. Furthermore, the neck volume is typically on the order of $5-10 \%$ of the total grain volume. Therefore, any errors caused by this assumption are small.

The mean surface area per unit volume can be derived by considering each of the grains as being detached from its neighbors and then subtracting from the surface area contained by the grain bonds. Underwood (1970) derived an expression for the surface area for a system of detached grains given by

$$
S=4 N_{\alpha L}
$$

Therefore, subtracting the area contained by the grain bonds from the above expression gives the correct surface area per unit volume

$$
S=4 N_{\propto L}-2 \pi r^{2} \bar{n}_{3} N_{\propto v} .
$$

\section{APPLICATION}

The theory presented above has been written into a computer code which uses a high-speed image analyzer located at Montana State University. The image analyzer is capable of digitizing a photograph of a section into approximately 300000 points and assigning each point one of 256 possibie gray levels. The digitized section is then imageenhanced and stored on a floppy disk for future analysis.

By using the image analyzer, exact point counts and extremely accurate length measurements can be made very quickly. This allows large areas of a section containing numerous grains to be analyzed which increases the statistical accuracy.

The following data are an example of the results of the theory using the image analyzer. A surface section was taken from a box of alpine snow stored in a cold room at $-12^{\circ} \mathrm{C}$ over the summer at Montana State University. Two areas of the section were then analyzed using the image analyzer. The areas of analysis are shown by the dashed and solid lines in Figure 1, respectively. The two areas overlap and hence are not statistically independent. However, the larger area does provide a check on the statistical accuracy of the smaller area. The data taken from the image analysis of the two areas are shown below. 
Area $1 \quad$ Area 2

\begin{tabular}{|c|c|c|}
\hline Area of analysis & $65.5 \mathrm{~mm}^{2}$ & $133.9 \mathrm{~mm}^{2}$ \\
\hline Ice-grain area fraction & 0.350 & 0.363 \\
\hline Ice-neck fraction & 0.038 & 0.043 \\
\hline Air-phase area fraction & 0.612 & 0.594 \\
\hline $\begin{array}{l}\text { Mean two-dimensional } \\
\text { bond length }\end{array}$ & $0.307 \mathrm{~mm}$ & $0.331 \mathrm{~mm}$ \\
\hline $\begin{array}{l}\text { Harmonic mean two- } \\
\text { dimensional bond length }\end{array}$ & $3.42 \mathrm{~mm}^{-1}$ & $3.30 \mathrm{~mm}^{-1}$ \\
\hline $\begin{array}{l}\text { Number of grains per unit } \\
\text { length of a random test } \\
\text { line }\end{array}$ & $0.803 \mathrm{~mm}^{-1}$ & $0.776 \mathrm{~mm}^{-1}$ \\
\hline
\end{tabular}

The data generated by the image analysis of the surface section are in good agreement for both areas analyzed. This indicates that the smaller area was representative of the overall properties of the section.

Using the above information, the two-dimensional theoretical probability distribution for grain coordination number can be computed. The results for each area analyzed along with the measured distributions are shown below.

No. of
bonds
$n=0$
$n=1$
$n=2$
$n>3$

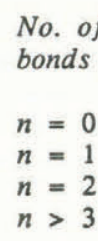

Area 1

$\begin{array}{cc}\begin{array}{c}\text { Measured } \\ \text { distribution }\end{array} & \begin{array}{c}\text { Theoretical } \\ \text { distribution }\end{array} \\ 0.518 & 0.515 \\ 0.291 & 0.313 \\ 0.139 & 0.102 \\ 0.052 & 0.070\end{array}$

Area 2

$\begin{array}{lcc}\begin{array}{l}\text { No. of } \\ \text { bonds }\end{array} & \begin{array}{c}\text { Measured } \\ \text { distribution }\end{array} & \begin{array}{c}\text { Theoretical } \\ \text { distribution }\end{array} \\ n=0 & 0.490 & 0.489 \\ n=1 & 0.327 & 0.327 \\ n=2 & 0.112 & 0.111 \\ n>3 & 0.071 & 0.073\end{array}$

The theoretical distributions for the number of bonds per grain, as seen in the section plane, compared very favourably with the measured distributions. For the larger area of analysis, the error is approximately $1 \%$ for grains with 0,1 , or 2 bonds, respectively. These grains account for approximately $93 \%$ of the grains in the section analyzed.

Using the theoretical data generated above in conjunction with the previous information determined by image analysis, the internal-state variables may be readily computed. These are given by the following:

$$
\text { Area } 2 \text { Area } 2
$$

Density ratio

Mean three-dimensional bond radius

\section{$0.230 \mathrm{~mm} \quad 0.238 \mathrm{~mm}$}

Mean three-dimensional bond length

$0.193 \mathrm{~mm} \quad 0.182 \mathrm{~mm}$

Mean intergranular slip distance

$0.762 \mathrm{~mm} \quad 0.766 \mathrm{~mm}$

Mean intercept length

$0.484 \mathrm{~mm} \quad 0.523 \mathrm{~mm}$

Mean number of bonds per grain

Mean number of grains per unit volume
Mean surface area per unit volume
$2.43 \mathrm{~mm}^{-1} 2.16 \mathrm{~mm}^{-1}$
The repeatability of the data for the internal-state variables for the two areas analyzed is quite strong with the difference between the two typically being on the order of $10 \%$ or less. Also, the density ratio determined by a field measurement of this particular snow sample was 2.29. This differs from the stereological measurements by $13 \%$ and $7 \%$, respectively for areas 1 and 2 . It is not surprising that the field-measured density was slightly higher than that determined by image analysis, since some compaction of the snow will occur when taking field data. Furthermore, etching of the ice grains during sectioning will cause the image analysis to predict a slightly lower density than the actual value.

\section{CONCLUSIONS}

A statistical model for snow based on internal-state variables of the "averaging" type has been developed. The model yields self-consistent results and is feasible to apply in a constitutive theory, since all of the parameters can be easily computed experimentally using image-analysis techniques.

A new stereological relation has been developed to characterize the mean three-dimensional length of a bond. This is a significant parameter for modeling viscous deformation.

The number of grains per unit volume and the number of bonds per grain, both of which are significant parameters in characterizing the granular structure of snow, are derived while making no assumptions on shape, size, or distribution of the grains. As a consequence of this, values for the mean surface area per unit volume, and mean volume of an ice grain are developed. All of these parameters will make significant contributions to a constitutive theory based on internal-state variables.

\section{ACKNOWLEDGEMENTS}

The work reported here was supported by the U.S. Army Research Office, grant No. DRXRO-18730-GA. The authors wish to express their appreciation to the U.S. Army Research Office for their support.

\section{REFERENCES}

Brown, R.L. 1979. A volumetric constitutive law for snow subjected to large strains and strain rates. CRREL Report 79-20.

DeHoff, R.T. 1964. Determination of the geometric properties of aggregates of constant-size particles from counting measurements made on random plane sections. Transactions of the Metallurgical Society of AIME, Vol. 230.

DeHoff, R.T. 1965. The estimation of particle-size distributions from simple counting measurements made on random plane sections. Transactions of the Metallurgical Society of $A I M E$, Vol. 233.

Fullman, R.L. 1953. Measurement of particle sizes in opaque bodies. Transactions of the Metallurgical Society of AIME, Vol. 197.

Gubler, H. 1978. Determination of the mean number of bonds per snow grain and of the dependence of the tensile strength of snow on stereological parameters. Journal of Glaciology, Vol. 20, No. 83, p. 329-41.

Hilliard, J.E. 1968. Direct determination of the moments of the size distributions of particles in an opaque sample. Transactions of the Metallurgical Society of AIME, Vol. 242.

Kry, P.R. 1975. Quantitative stereological analysis of grain bonds in snow. Journal of Glaciology, Vol. 14, No. 72, p. 467-77.

Underwood, E.E. 1970. Quantitative stereology. Reading, MA, Addison-Wesley Publishing Co. 